\title{
ÉTUDE DE LA CLASSIFICATION DES SOUFFRANCES À DISTANCE DE LUC BOLTANSKI
}

\section{Christian Bergeron, Ph.D. \\ Université Laval}

\begin{abstract}
Cette étude porte sur la perception qu'ont les adultes des changements survenus dans leur vie et dans la société. Selon une méthodologie mixte de recherche, 504 adultes de la Ville de Québec ont participé à notre étude doctorale. Dans le cadre de cet article, nous nous intéressons à la notion de la souffrance à distance de Luc Boltanski, c'est-à-dire la souffrance des autres qui s'intègre dans le soi historique des individus. L'analyse quantitative, des événements et changements sociohistoriques marquants pour les participants, montre l'importance des événements terroristes du 11 septembre 2001, suivis des référendums sur l'indépendance du Québec. L'analyse de contenu, centrée sur le vécu et la gestion des souffrances à distance, valide la portée heuristique de la classification de Boltanski.

Mots-clés: Souffrance à distance ; Luc Boltanski ; soi historique ; 11 septembre 2001; Haïti

This study focuses on adults' perception of changes in their lives and in society. Following a mixed research methodology, 504 adults in Quebec City participated in our doctoral study. In the context of this article, we focus on the notion of distant suffering by Luc Boltanski, i.e., the suffering of others that fits into the historical self of individuals. Quantitative analysis of sociohistorical events and changes significant for the participants shows the extent of the effects of the terrorist events of September 11, 2001, followed by the referendums on Quebec independence. Content analysis, focusing on the experience and management of distant suffering, validates the heuristic classification of Boltanski.
\end{abstract}

Keywords: distant suffering; Luc Boltanski; historical self; September $11^{\text {th }} 2001$; Haiti.

\section{Introduction}

Dans le cadre de notre recherche doctorale, nous étudions la société québécoise inscrite dans la modernité avancée, telle que présente dans les pays occidentaux. Nous portons notre regard sur les événements collectifs jugés marquants et qui viennent introduire une rupture, une discontinuité, dans les parcours de vie individuels et collectifs. Par exemple, certains événements et changements sociohistoriques seront vécus comme des épreuves collectives, telles qu'une guerre, une crise économique, un bouleversement politique, une catastrophe naturelle, alors que d'autres ne seront pas interprétés comme des épreuves collectives, telles des avancées technologiques (télévision, routes, moyens de transport, informatique...), des victoires politiques ou sportives. Notre analyse cible les événements et les changements sociohistoriques interprétés par les individus en termes d'épreuve à proximité de soi et celle à distance (une épreuve vécue par des malheureux; cf. Boltanski, 1993). L'épreuve à proximité de soi est une épreuve collective vécue individuellement ou éprouvée par d'autres, malheureux, mais individualisée et intégrée au soi historique (développement de la conscience collective; cf. Mannheim, [1928] 1990). 
En raison de l'absence d'écrits scientifiques et sociologiques sur les épreuves collectives, mais surtout de leur compréhension par l'entremise de la classification de Boltanski (1993), il devient original et pertinent de les étudier dans le cadre de notre recherche. Les sociologues ont peu d'instruments de mesure pour étudier et comprendre la formation du soi historique ainsi que sa signification. En outre, à notre connaissance, aucune étude n'a validé la classification de Boltanski (1993) en lien avec le soi historique. Cela dit, nous avons repéré une seule étude qui a étudié cette classification auprès de détenus français (Chabbal, 2009). L'objectif de cette étude est de repérer des épreuves contemporaines collectives et de valider la pertinence scientifique de la classification de Boltanski sur la souffrance des autres (épreuves à proximité de soi et à distance).

\section{L'identité narrative et le soi historique}

Lorsqu'un individu se raconte, à savoir qui a fait quoi, comment et pourquoi, il se construit une unité dans l'action, où d'autres individus viennent s'inscrire dans son récit autobiographique grâce à l'enchevêtrement d'un tissu d'histoires racontées par lui-même et celles des autres (Ricœur, 2005). Ce récit autobiographique définit l'identité narrative. Plus précisément, l'identité narrative lie la capacité individuelle à organiser, structurer un récit sur soi-même, à la possibilité de se rattacher à une histoire dans laquelle l'individu se reconnaît et s'alimente (Ricœur, 1990). C'est une histoire individuelle qui est racontée «avec ses caractères d'unité, d'articulation interne et de complétude, conférés par l'opération de mise en intrigue, que le personnage conserve tout au long de l'histoire une identité corrélative de celle de l'histoire elle-même » (Ricœur, 1990, p. 170). De cette manière, le récit dialectique construit l'identité narrative et, par le fait même, construit l'histoire : «chacun se raconte l'histoire de sa vie qui donne sens à ce qu'il vit » (Kaufmann, 2004, p. 152). C'est en effet un travail discursif «par lequel l'individu parvient à se doter d'une représentation unitaire et cohérente de soi » (Martuccelli, 2002, p. 367).

En résumé, l'identité se forge à travers un récit narré par l'individu (sélection subjective d'événements de la vie) et à travers le temps (travail permanent et de maintien de repères). L'individu se définit ainsi : « je suis ce que je me raconte » (Deschavanne et Tavoillot, 2007, p. 265 ) «dans un récit de vie à la fois intelligible pour [ma] raison et acceptable pour [mon] cœur » (Ricœur, 2005, p. 2). L'individu recherche une cohérence interne de lui-même (unicité de l'identité par délimitation) en interpénétration et appartenance avec le monde social, les différents «nous » (processus d'équilibrage continuel). L'intégration des différents «nous » (cf. épreuve à proximité de soi) et « eux » (cf. épreuve à distance) dans l'identité narrative peuvent se comprendre à l'aide du soi historique (conscience historique). Le soi historique « est la façon dont certains individus se comprennent eux-mêmes comme un produit de l'histoire et en même temps comprennent le sens liminaire de l'histoire à l'échelle de leurs vies » (Martuccelli, 2006, p. 236). De fait, le soi historique se construit en prenant conscience d'événements sociohistoriques (en ce qui nous intéresse, la souffrance individuelle et celle des autres), intégrés dans l'identité narrative.

\section{L'épreuve à distance et l'épreuve à proximité de soi}

La notion de l'épreuve à distance s'inspire de celle de la souffrance à distance développée par Boltanski (1993) et qui s'inscrit dans l'esprit de la Théorie des sentiments moraux d'Adam Smith ([1759] 2003). Certains événements sociohistoriques, tragiques et malheureux, peuvent être 
compris comme des épreuves collectives vécues par des étrangers. Plus particulièrement, c'est «l'observation des malheureux par ceux qui ne partagent pas leurs souffrances, qui n'en ont pas l'expérience directe, et qui peuvent, à ce titre, être considérés comme des gens heureux » (Boltanski, 1993, p. 16). Notre regard est porté non pas sur les malheureux ayant vécu l'épreuve, mais sur celui qui en prend connaissance et qui le met en dialogue avec son soi historique. Nous étudions également les épreuves à proximité, c'est-à-dire les épreuves collectives que l'individu a vécues lui-même ou éprouvées par des malheureux, mais qui a intégré la souffrance des autres dans son soi historique. En quelque sorte, l'individu devient un acteur («identification-à ») de l'événement en compagnie d'autres contemporains; il n'est plus un spectateur, puisqu'il observe les conséquences directes ou indirectes de l'événement dans sa vie. Par exemple, certains événements malheureux, comme la crise d'octobre de 1970 et le grand verglas de janvier 1998 au Québec, peuvent se classer comme des épreuves à proximité pour plusieurs personnes.

Abordons plus en détail la notion d'épreuve à distance. Les analyses de Boltanski, sur la souffrance à distance, ont mis en évidence trois actions possibles de l'individu lorsqu'il prend connaissance de l'épreuve des autres : 1) de l'indignation à l'accusation, 2) la gratitude du malheureux et 3) le sentiment de l'urgence.

De l'indignation à l'accusation. - Ces actions s'inscrivent dans une topique de la dénonciation. «Face au spectacle d'un malheureux souffrant au loin, que peut faire un spectateur, condamné au moins dans l'immédiat —, à l'inaction mais moralement bien disposé? Il peut s'en indigner » (Boltanski, 1993, p. 91). L'indignation ou même la révolte engendrée par une épreuve à distance peuvent passer par un sentiment de pitié. "Mais dans l'indignation, la pitié est transformée. Elle ne demeure pas désarmée et, par conséquent, impuissante, mais se dote des armes de la colère » (Boltanski, 1993, p. 91). L'individu devant une épreuve à distance, peut passer du sentiment de l'indignation à la colère, puis à l'accusation d'un coupable. Le sentiment de pitié, devant un spectacle désolant, peut réorienter l'attention de l'individu (du spectateur et des acteurs) vers la recherche d'un ou des responsables de ces malheurs. Par conséquent, l'individu veut éliminer ce sentiment d'indignation et se détourne rapidement de l'événement catastrophique des malheureux, vers l'extérieur, vers l'identification d'un «persécuteur». Pour faire suite aux inondations meurtrières de 2007 et surtout celles de 2008 (quatre cyclones) en Haïti, certains analystes et représentants d'organisations non gouvernementales (p. ex.: Greenpeace) ont ciblé les constructions en zones inondables et la déforestation accélérée pour expliquer ce drame humanitaire. Le spectateur prenant connaissance de ces inondations peut accuser un système étatique corrompu et le juger injuste à l'égard de ses citoyens. L'indignation (colère, sentiment d'injustice), devant un drame d'une telle ampleur, éclate et se transpose dans différents actes d'accusation et de dénonciation.

La gratitude du malheureux. - Ici, l'individu ne développe pas un sentiment d'indignation qui le conduit à l'accusation, mais il éprouve de la reconnaissance, un sentiment de gratitude, à l'égard de ceux qui viennent en aide aux malheureux. L'action n'est pas engagée vers la recherche d'un persécuteur, mais bien d'un bienfaiteur (Boltanski, 1993). En d'autres mots, l'épreuve à distance amène un sentiment de pitié et oriente l'attention du spectateur «vers la possibilité d'une bienfaisance accomplie par un bienfaiteur » (Boltanski, 1993, p. 117). L'individu est attendri par l'action des bienfaiteurs qui viennent en aide aux malheureux. En janvier 2010, Haïti a été victime de violents séismes meurtriers et dévastateurs. Devant ce nouveau drame humanitaire, certains 
individus ont emprunté le chemin de l'indignation vers l'accusation (colère et injustice) alors que d'autres ont exprimé leur pitié dans un sentiment de reconnaissance provoqué par les actes de bienfaisances accomplis par des bienfaiteurs (aide humanitaire et monétaire de certains pays). D'une certaine manière, le sentiment de gratitude à l'égard d'un bienfaiteur peut découler de la perception de corriger une injustice humanitaire. En somme, la gratitude du malheureux conduit différemment à l'action.

Le sentiment de l'urgence. - La pitié peut aussi emprunter un autre chemin, celui de l'urgence d'agir pour l'individu : ce dernier veut lui-même venir en aide aux malheureux. La souffrance éprouvée par l'épreuve à distance ne peut attendre, d'où l'urgence d'agir pour l'individu (Boltanski, 1993). Ce dernier peut s'engager directement dans l'action. Par exemple, les médecins et le personnel médical canadien sont venus prêter secours aux sinistrés haïtiens ou encore, le spectateur qui a réagi rapidement en faisant un don d'argent à la Croix-Rouge canadienne. C'est maintenant plus qu'un sentiment de gratitude (du bienfaiteur) ou de colère (du persécuteur) : l'individu devient impliqué dans le déroulement de l'épreuve de l'autre, du malheureux.

L'individu qui prend connaissance d'une épreuve à distance «n'est pas plongé dans la situation où se trouve le malheureux; il n'est pas à ses côtés pendant son agonie ou son supplice » (Boltanski, 1993, p. 221). Le sort du malheureux doit alors susciter ou éveiller de la sympathie chez le spectateur pour qu'il s'engage dans l'une des trois formes d'action (Boltanski, 1993). Des sentiments (indignation, accusation, gratitude, urgence) doivent s'exprimer dans une nonindifférence à l'égard de ce malheureux. Précisons également que ces trois formes d'action vis-àvis de l'épreuve à distance peuvent coexister. Reprenons l'exemple d'Haïti 2010. Un individu peut simultanément s'indigner et cibler un coupable tout en donnant une somme d'argent à la CroixRouge. D'ailleurs, ces mêmes actions et sentiments peuvent se trouver dans un événement local, dans une épreuve à proximité. Par exemple, la crise du verglas au Québec a inspiré différentes réactions : certains gens ont été poussé à l'action, d'autres se sont émus de l'entraide et un certain nombre de personnes ont exprimé un sentiment de colère envers les autorités. Cela dit, est-ce l'acte accusatoire, de gratitude ou d'urgence qui s'intègre plus aisément dans le soi historique des individus? Pour répondre à cette question et bien saisir l'importance des épreuves vécues par des étrangers ou par des concitoyens, il faut se référer au soi historique, c'est-à-dire à la conscience individuelle d'événements sociohistoriques. Les épreuves à distance et celles à proximité de soi seront explorées avec le soi historique lors des analyses mixtes des résultats.

\section{Méthodologie de recherche}

Notre recherche s'intègre dans l'étude internationale Changements et événements au cours de la vie (CEVI). Elle scrute le déroulement des vies individuelles dans leur relation avec le contexte sociétal et historique, en prenant pour étude la notion d'événement marquant de la vie ainsi que la perception qu'en a l'individu. Dans le cadre de cet article, nous présentons uniquement les résultats de l'étude canadienne en lien avec l'étude de la classification de Boltanski.

Nous utilisons le questionnaire standardisé de l'étude CEVI (cf. Lalive d'Epinay, et coll., 2008). La troisième section de l'enquête nous est utile pour comprendre l'intégration de la souffrance, individuelle et celle des autres, dans les différents soi historique des individus. La question posée 
est celle-ci : «Considérons maintenant les grands événements et changements qui se sont produits dans votre pays (ou votre province) et dans le monde au cours de votre vie. Quels sont ceux qui vous ont le plus frappés? » La personne interrogée doit en faire une description et ensuite répondre à la question : «Pourquoi vous a-t-il marqué particulièrement? » La personne interrogée peut mentionner jusqu'à quatre événements et changements sociohistoriques et aussi préciser l'année, l'âge et le lieu au moment du changement.

Afin de pouvoir effectuer des études comparatives, nous avons utilisé une méthodologie de la recherche similaire aux autres équipes CEVI. L'objectif est d'atteindre de 500 à 600 participants comme dans les autres études internationales. Dans notre recherche canadienne, nous avons obtenu la participation de 504 personnes de l'agglomération urbaine de la Ville de Québec. L'échantillon de l'étude, non-aléatoire, est stratifié selon le sexe et selon cinq classes d'âges quinquennales séparées par un intervalle de 10 ans : 20-24, 35-39, 50-54, 65-69 et 80-84 ans qui représentent des cohortes d'individus nés entre 1926 et 1990.

\section{La méthode mixte de recherche}

Le choix d'une méthode mixte de recherche implique la réalisation de quatre phases : 1) la mise en œuvre, 2) la priorisation, 3) l'intégration et 4) la perspective théorique (cf. Creswell, 2003). Pour la mise en ouvre, nous n'avons qu'une phase de recrutement, nous utilisons donc un mode de collecte de données en simultané. Deuxièmement, les données quantitatives sont priorisées en prévision des phases suivantes (codification préétablie des questions ouvertes du questionnaire). Ensuite, nous procédons à une deuxième codification des données quantitatives pour l'identification des épreuves collectives (épreuves à proximité de soi et à distance). Troisièmement, nous utilisons une catégorisation de l'analyse de contenu dirigée tirée de la classification de Boltanski. Quatrièmement, la perspective théorique, la classification des épreuves à distance et à proximité de soi, guide l'interprétation des événements et changements sociohistoriques. Enfin, pour identifier la classification de Boltanski, nous avons élaboré des indices et des indicateurs.

Le repérage des indices et l'élaboration d'indicateurs de l'épreuve à proximité de soi et à distance. - Les épreuves vécues à proximité de soi sont essentiellement des changements sociohistoriques auxquels l'individu a participé ou encore a le sentiment d'avoir subi les conséquences. En d'autres mots, l'épreuve à proximité est une épreuve collective vécue individuellement ou éprouvée par d'autres, malheureux, mais individualisée et intégrée au soi historique. Pour les épreuves à distance, nous empruntons à Boltanski (1993) sa classification portant sur les émotions et les sentiments suscités par un événement sociohistorique. Les indices de classification sont l'expression d'un sentiment : 1) d'indignation, 2) de gratitude et 3) d'urgence (passage à l'action). Cette classification permet d'étudier les manières dont les individus individualisent des événements et des changements sociohistoriques jugés comme des épreuves collectives. Précisons que certains participants ont cité des épreuves collectives sans pour autant exprimer d'émotions ou des sentiments qui se référent à des épreuves explicitement. De plus, certaines explications étaient trop brèves pour identifier les indices et les codifier d'épreuves collectives.

En résumé, notre questionnaire, composé de questions ouvertes (phase I), favorise une nouvelle codification de nos données quantitatives (phase II) pour l'identification des épreuves collectives. La méthode quantitative est priorisée pour leur identification, car «les chiffres sont plus 
économiques et manipulables que les mots; on «voit» plus vite et plus facilement la tendance générale des données en examinant leur distribution ». (Huberman et Miles, 1991, p. 385). Ensuite, l'analyse de contenu (phase III) nous guide dans la catégorisation et l'identification de la classification de Boltanski en prévision de leur interprétation avec notre approche théorique (phase IV).

\section{Résultats}

En 2010, 504 participants de la Ville de Québec ont participé à notre étude. Excepté les octogénaires (50\% d'entre eux vivent seuls), les participants cohabitent avec un partenaire ou un colocataire (de $48 \%$ à $73 \%$ ). Aussi, ils sont majoritairement parents d'au moins un enfant (de $59 \%$ à $78 \%$ ), à l'exception des personnes de 20 à 24 ans (2\%). Ils sont davantage scolarisés que la population québécoise. Chez les 20-24 ans et les 35-39 ans, 9 participants sur 10 ont une scolarité supérieure ou égale au niveau collégial ou universitaire, alors qu'au Québec c'est seulement 5 individus sur 10 (45 \%) (Statistique Canada, 2006). En outre, chez les 80-84 ans, 4 participants sur 10 ont une scolarité supérieure ou égale au niveau collégial ou universitaire, alors que $27 \%$ des 65 ans et plus ont la même scolarité au Québec.

Tableau 1

Les événements et changements sociohistoriques les plus fréquents par classes d'âges

\begin{tabular}{|c|c|c|c|c|c|c|c|c|c|}
\hline \multicolumn{2}{|c|}{ 20-24 ans } & \multicolumn{2}{|c|}{$35-39$ ans } & \multicolumn{2}{|l|}{$50-54$ ans } & \multicolumn{2}{|l|}{$65-69$ ans } & \multicolumn{2}{|c|}{$80-84$ ans } \\
\hline Type & $\%$ & Type & $\%$ & Type & $\%$ & Type & $\%$ & Type & $\%$ \\
\hline 11 sept 01 & 29 & 11 sept 01 & 22 & 11 sept 01 & 15 & $\begin{array}{l}\text { Élection du } \\
\text { PQ } 76\end{array}$ & 8 & $2^{\text {ième }} \mathrm{GM}$ & 24 \\
\hline $\begin{array}{l}\text { Référendum } \\
95\end{array}$ & 8 & $\begin{array}{l}\text { Référendum } \\
95\end{array}$ & 11 & $\begin{array}{l}\text { Élection du } \\
\text { PQ } 76\end{array}$ & 8 & 11 sept 01 & 7 & $\begin{array}{l}\text { Révolution } \\
\text { Tranquille }\end{array}$ & 7 \\
\hline $\begin{array}{l}\text { Élection } \\
\text { Obama } 08\end{array}$ & 4 & $\begin{array}{l}\text { Progrès } \\
\text { comm.* }\end{array}$ & 5 & $\begin{array}{l}\text { Référendum } \\
80\end{array}$ & 6 & $\begin{array}{l}\text { Progrès } \\
\text { comm. }\end{array}$ & 6 & $\begin{array}{l}\text { Progrès } \\
\text { comm. }\end{array}$ & 5 \\
\hline $\begin{array}{l}\text { Tempête de } \\
\text { Verglas } 98\end{array}$ & 4 & $\begin{array}{l}\text { Élection } \\
\text { Obama } 08\end{array}$ & 4 & $\begin{array}{l}\text { Crise octobre } \\
70\end{array}$ & 6 & Mort JFK & 6 & $\begin{array}{l}\text { Élection du } \\
\text { PQ } 76\end{array}$ & 4 \\
\hline Haïti 10 & 4 & Haïti 10 & 4 & $\begin{array}{l}\text { Homme sur la } \\
\text { lune }\end{array}$ & 4 & $\begin{array}{l}\text { Crise octobre } \\
70\end{array}$ & 6 & 11 sept 01 & 3 \\
\hline Autres & 51 & Autres & 54 & Autres & 61 & Autres & 67 & Autres & 57 \\
\hline Total & 100 & Total & 100 & Total & 100 & Total & 100 & Total & 100 \\
\hline
\end{tabular}

$(\chi 2=1058,94, p \leq 0,001)$

* Progrès informatiques et communications 
Le tableau 1 présente les événements et changements sociohistoriques les plus mentionnés pour les cinq classes d'âges. L'événement le plus cité est celui du 11 septembre 2001, et ce, chez les trois plus jeunes classes d'âges $(29 \%, 22 \%, 15 \%$ respectivement) et en deuxième chez les 65-69 ans (7\%). Toutefois, cet événement est presque absent chez les participants de 80-84 ans (3\%). Attirons l'attention sur les deux référendums sur la souveraineté du Québec. Nous remarquons que le dernier référendum de 1995 est quasiment inexistant chez les cohortes âgées de plus de 50 ans (les 50-54 ans : 8 mentions; les 65-69 ans : 5 mentions; et les 80-84 ans : une mention). Également, la mention du premier référendum de 1980 est absente de chez les participants de 65 ans et plus. C'est uniquement l'élection de René Lévesque, en 1976, qui est mentionnée comme événement frappant pour les groupes de 50 ans et plus. Finalement, une grande diversité des changements sociohistoriques est mentionnée chez les participants, comme en témoigne la catégorie "Autres » (de $51 \%$ à $67 \%$ des événements cités).

\section{Résultats de la classification de Boltanski}

Les épreuves à proximité de soi et à distance sont codifiées à partir d'événements et de changements sociohistoriques cités par les répondants. Boltanski, avec la souffrance à distance, catégorise trois actions possibles de l'individu lors de l'épreuve des autres: 1) de l'indignation à l'accusation. L'individu s'approprie l'épreuve et exprime un sentiment de colère (dénonciation et accusation) dans la recherche d'un coupable, 2) la gratitude du malheureux. L'individu est attendri par l'action des bienfaiteurs qui viennent en aide aux malheureux et 3) le sentiment de l'urgence. La personne s'engage dans une action d'urgence; c'est plus qu'un sentiment de gratitude (envers le bienfaiteur) ou de colère (envers le persécuteur), car elle veut agir et être plus qu'une spectatrice de l'épreuve du malheureux. Nous avons ajouté à cette classification, la catégorie «épreuve à proximité », rencontrée lorsqu'un individu n'exprime pas de la pitié à distance au sens de Boltanski, mais un sens personnalisé de proximité avec une épreuve. En guise d'exemple, les attentats du 11 septembre 2001 sont venus complexifier les voyages pour plusieurs, notamment ceux prévus aux États-Unis. En outre, certains participants, qui ont de la famille ou des amis à New York, se sont sentis davantage concernés par ces événements terroristes. À titre d'exemple de cette épreuve à proximité, citons un participant : "mon père était au New Jersey pendant l'évènement et nous étions tous très inquiets ».

Le tableau 2 répertorie les types d'épreuves conformément à la classification de Boltanski par classes d'âges. Il est pertinent de mentionner qu'il existe des différences significatives entre ces types d'épreuves. Prenons uniquement les épreuves à distance. Elles sont mentionnées chez les 2024 ans (25\%), déclinent graduellement à travers les cohortes (20\% chez les 35-39 ans; $15 \%$ chez les 50-54 ans; $10 \%$ chez les 65-69 ans) pour terminer à $6 \%$ chez les 80-84 ans. Cependant, les changements sociohistoriques, qui ne sont pas des épreuves, suivent le chemin inverse : $47 \%$ des classes plus âgées ne les mentionnent pas comme des épreuves collectives. De plus, ce pourcentage décline graduellement jusqu'à $26 \%$ chez les 20-24 ans.

Nous remarquons également qu'il y a très peu d'épreuves collectives qui s'intègrent totalement à la classification de Boltanski. Ce sont uniquement les sentiments d'indignation ou de colère qui semblent s'exprimer à travers celles-ci. Il n'y a pratiquement aucune de ces épreuves qui conduisent les participants vers l'action et très peu vers un sentiment de gratitude. Toutefois, il existe un cas d'exception, soit le tremblement de terre du 12 janvier 2010 en Haïti (nous y reviendrons lors de la 
discussion). Le tableau 3 présente les principaux événements et changements sociohistoriques selon notre classification des épreuves collectives. Parmi les épreuves à proximité : la Seconde Guerre mondiale est l'événement le plus cité (77\%), suivi de la crise d'octobre de 1970 au Québec (73\%) et de l'assassinat de John F. Kennedy (JFK) en 1963 à Dallas (53\%).

Tableau 2

Distribution des types d'épreuves selon la classification de Boltanski par classes d'âges

\begin{tabular}{|c|c|c|c|c|c|c|c|c|c|}
\hline $20-24$ ans & & 35-39 ans & & $50-54$ ans & & $65-69$ ans & & $80-84$ ans & \\
\hline Épreuve & $\%$ & Épreuve & $\%$ & Épreuve & $\%$ & Épreuve & $\%$ & Épreuve & $\%$ \\
\hline À proximité & 31 & $\begin{array}{l}\text { Pas une } \\
\text { épreuve }\end{array}$ & 34 & $\begin{array}{l}\text { Pas une } \\
\text { épreuve }\end{array}$ & 44 & $\begin{array}{l}\text { Pas une } \\
\text { épreuve }\end{array}$ & 47 & $\begin{array}{l}\text { Pas une } \\
\text { épreuve }\end{array}$ & 47 \\
\hline $\begin{array}{l}\text { Pas une } \\
\text { épreuve }\end{array}$ & 26 & À proximité & 31 & À proximité & 27 & À proximité & 27 & À proximité & 33 \\
\hline À distance & 25 & À distance & 20 & À distance & 15 & À distance & 10 & À distance & 6 \\
\hline Indignation & 20 & Indignation & 16 & Indignation & 14 & Indignation & 9 & Indignation & 6 \\
\hline Gratitude & 3 & Gratitude & 1 & Gratitude & 1 & Gratitude & 1 & Gratitude & 0 \\
\hline Action & 2 & Action & 3 & Action & 0 & Action & 0 & Action & 0 \\
\hline Autre & 18 & Autre & 15 & Autre & 14 & Autre & 16 & Autre & 14 \\
\hline Total & 100 & Total & 100 & Total & 100 & Total & 100 & Total & 100 \\
\hline
\end{tabular}

Nommément, le génocide au Rwanda (67\%), la guerre en Irak (60 \%) et le tremblement de terre en Haïti (52\%) sont les épreuves à distance les plus importantes qui ont suscité des sentiments d'indignation ou de colère. Trois événements inscrits dans le tableau 3 méritent d'être soulignés, soit le 11 septembre 2001 et les deux référendums québécois sur l'indépendance du Québec. D'une part, les deux référendums (1980 et 1995) semblent être à l'image de la pensée actuelle du Québec : une certaine polarisation des positions idéologiques et politiques sur l'interprétation de ces événements sociohistoriques. Pour certains (42\%), le référendum de 1995 a été une épreuve (échec devant la possibilité d'avoir un pays, tristesse, sentiment de trahison et de vol, etc.). Un homme de 65 ans souligne que cette défaite référendaire a entraîné une : "désillusion et perte d'espérance de voir le Québec devenir un pays de [son] vivant», alors que pour $50 \%$ des participants, le référendum de 1995 n'a pas été une épreuve (demeurer dans le Canada, ne pas subir l'indépendance du Parti Québécois, garder son emploi, etc.). À cet effet, une dame de 80 ans mentionne : "parce que je suis contre et qu'on perd notre temps. Mes expériences en Europe m'ont ouvert les yeux. » D'autre part, autant de participants citent les attentats terroristes en 2001 comme une épreuve à proximité de soi (36\%) qu'une épreuve à distance avec indignation $(38 \%)$. Une certaine forme d'ambivalence semble exister dans l'appropriation de cet acte terroriste dans leur vie personnelle. 
Tableau 3

Distribution de certains événements et changements sociohistoriques selon l'épreuve (\% en colonne)

\begin{tabular}{lllllll}
\hline Événement & $\begin{array}{l}\text { Épreuve à } \\
\text { proximité }\end{array}$ & $\begin{array}{l}\text { Épreuve à } \\
\text { distance }- \\
\text { indignation }\end{array}$ & $\begin{array}{l}\text { Autre } \\
\text { épreuve à } \\
\text { distance }\end{array}$ & $\begin{array}{l}\text { Pas une } \\
\text { épreuve }\end{array}$ & $\begin{array}{l}\text { Épreuve sans } \\
\text { classement** }\end{array}$ & $\begin{array}{l}\text { Total } \\
(\%)\end{array}$ \\
\hline $2^{\text {ième }}$ GM & 77 & 4 & 4 & 1 & 17 & 100 \\
Crise octobre 70 & 73 & 2 & 1 & 2 & 22 & 100 \\
Assassinat JFK & 52 & 7 & 3 & 7 & 33 & 100 \\
Référendum 95 & 42 & 0 & 1 & 50 & 7 & 100 \\
11 septembre 01 & 36 & 38 & 3 & 0 & 23 & 100 \\
Référendum 80 & 27 & 0 & 0 & 53 & 20 & 100 \\
Génocide au & 22 & 67 & 0 & 0 & 11 & 100 \\
Rwanda & & & & & & 100 \\
Haïti 10 & 15 & 52 & $30 *$ & 0 & 3 & 100 \\
Guerre en Irak & 6 & 60 & 1 & 13 & 20 & \\
\hline
\end{tabular}

* Ce pourcentage inclut $21 \%$ pour un sentiment de gratitude et $9 \%$ pour l'urgence d'agir (action).

** Les explications des répondants ne permettaient pas de les classer dans une catégorie d'épreuve.

\section{Discussion}

Pour l'ensemble des participants, les événements et changements sociohistoriques les plus cités sont les événements du 11 septembre $2001(15 \%)$, la Deuxième Guerre mondiale $(5 \%)$, les progrès informatiques (communications) (5\%) ainsi que le référendum de 1995 sur la souveraineté du Québec (5\%; cf. tableau 1). Selon nos analyses mixtes (avec la classification des épreuves à proximité de soi et à distance), les participants de 50 ans et plus citent, davantage que les cohortes plus jeunes, des événements et changements sociohistoriques qui ne sont pas catégorisés comme des épreuves collectives (50-54 ans : $44 \%$; 65-69 ans et 80-84 ans: $47 \%$; cf. tableau 2). Inversement, chez les cohortes des 20-24 ans et des 35-39 ans, les épreuves à proximité de soi sont les plus citées (31\%; cf. tableau 2). En effet, la Deuxième Guerre mondiale (77\%), la crise d'octobre de 1970 au Québec (73\%) ainsi que l'assassinat de JFK en 1963 à Dallas, sont les épreuves à proximité les plus citées (53\%; cf. tableau 3). Notons que plus les participants sont âgés, moins ils mentionnent une épreuve à distance (20-24 ans : 25\%;35-39 ans : 20\%; 50-54 ans : $15 \%$; 65-69 ans : $10 \%$ et 80-84 ans : $6 \%$ ). Il semble que la classification des sentiments à distance de Boltanski se vérifie davantage auprès des jeunes cohortes. Les types d'événements sociohistoriques cités influencent certainement l'expression ou non des sentiments à distance. Par exemple, nous savons que les événements terroristes du 11 septembre 2001 sont davantage cités par les jeunes cohortes et qu'ils sont classés, ex ego, comme une épreuve à proximité de soi (36\%) et comme une épreuve à distance avec indignation (38\%; cf. tableau 3). De plus, le tremblement de terre en Haïti, le génocide au Rwanda ainsi que la guerre en Irak sont majoritairement interprétés comme des épreuves à distance $(52 \%, 67 \%$ et $60 \%$ respectivement). Ces tragédies sont majoritairement mentionnées par les cohortes plus jeunes. 
Nous remarquons qu'il y a très peu d'épreuves collectives qui s'intègrent en totalité à la classification des épreuves à distance de Boltanski. Ce sont essentiellement les sentiments d'indignation ou de colère qui semblent s'exprimer à travers celles-ci. Précisons que

La transformation de la pitié en indignation suppose précisément une réorientation de l'attention, qui se détourne de la considération déprimante du malheureux et de ses souffrances pour aller chercher un persécuteur et se centrer sur lui (Boltanski, 1993, p. 91).

Selon nos analyses mixtes, il n'y a pratiquement aucune de ces épreuves étudiées qui conduisent les participants vers l'action et très peu vers un sentiment de gratitude. Toutefois, il existe un cas d'exception, soit le tremblement de terre du 12 janvier 2010 en Haïti. En se référant au tableau 3, cet événement est interprété comme une épreuve à proximité pour $15 \%$ des répondants (p. ex. : «j'ai une amie qui travaille là comme travailleuse humanitaire. J'avais peur pour elle », une femme âgée de 35 ans). De plus, $52 \%$ d'entre eux expriment un sentiment d'indignation (p. ex. : «situation sans issue. Pauvreté et dénuement du peuple. Régime corrompu, pas d'espoir», une femme âgée de 55 ans) et $21 \%$ éprouvent un sentiment de gratitude (p. ex. : "énormément de personnes vont aider ce pays en péril et cela me touche beaucoup », une femme âgée de 20 ans). Finalement, $9 \%$ des participants s'engagent dans une action individuelle (p. ex. : «j'ai envoyé des dons et de la nourriture pour aider les sinistrés», dit un homme âgé de 36 ans). En somme, la classification de Boltanski s'observe à travers cette catastrophe d'envergure qui a causé plus de 230000 morts, 300000 blessés et plus de 1200000 sans-abris (Radio-Canada, 2010). L'ampleur du drame, qui a provoqué des émotions et des sentiments d'impuissance ou d'injustice, a peut-être favorisé le repérage de cette classification, à tout le moins chez les cohortes de moins de 40 ans. L'émotion médiatique est réelle avec cette catastrophe humanitaire, c'est-à-dire qu' « elle se rattache aux émotions réelles dans la mesure où la souffrance des malheureux donnée en spectacle est présentée comme réelle, proposée à un mode d'adhésion relevant de la croyance existentielle, son authenticité pouvant faire l'objet d'un jugement »(Boltanski, 1993, p. 221). Ainsi, nous pouvons affirmer que le spectateur canadien, devant l'authenticité de la souffrance des Haïtiens, a éprouvé des émotions réelles qui se sont traduites dans l'expression des topiques de la souffrance des autres.

En conclusion de la discussion, il est pertinent de soulever un biais méthodologique. Le recrutement des participants a démarré quelques jours après les événements tragiques en Haïti. Selon nous, il est fort probable que certains participants ont été influencés par cette catastrophe au moment de répondre au questionnaire de l'étude. Il semble que les cohortes d'âges les plus jeunes ont été davantage influencées, voire impressionnées par l'événement. D'autres études seront nécessaires afin de déterminer, d'une part, si le tremblement de terre en Haïti demeure dans le soi historique des individus et d'autre part, si la classification de Boltanski permet de mettre en lumière les sentiments associés à de nouveaux événements sociohistoriques. D'ailleurs, il serait intéressant que d'autres équipes canadiennes valident cette classification afin de comparer les résultats et d'ainsi améliorer la compréhension du développement du soi historique et, globalement, de la mémoire collective. 


\section{Conclusion}

La compréhension de la notion d'épreuve, par l'entremise de la classification des sentiments à distance de Boltanski, a permis de mieux comprendre comment les individus s'approprient la souffrance des autres dans leur soi historique. La classification de Boltanski sur la souffrance des autres est pertinente pour l'étude des mémoires collectives dans un contexte sociohistorique de globalisation. En d'autres mots, la souffrance des autres devient une épreuve à distance qui s'intègre dans le corpus de la mémoire collective d'une nation ou de plusieurs pays. Le Québec n'est pas différent des autres sociétés occidentales, car les événements internationaux s'intègrent dans le soi historique des individus, notamment chez les jeunes adultes. L'instantanéité des nouvelles nationales et internationales ainsi que l'importance des médias sociaux influencent certainement les nouvelles manières contemporaines de construire sa conscience historique à partir d'événements et changements sociohistoriques jugés frappants dans (et pour) le monde. Par exemple, d'autres études confirment l'importance de la globalisation chez la mémoire historique des jeunes, notamment considérant l'importance pour eux des événements terroristes du 11 septembre 2001 (Bergeron, 2015; Lalive d'Epinay et coll., 2012). À long terme, la souffrance des autres, véhiculée par les médias sociaux, prendra peut-être beaucoup plus d' " espaces » dans les différentes mémoires collectives.

\section{Remerciements}

Ces résultats sont tirés de ma thèse de doctorat en sociologie. J'ai obtenu une bourse d'excellence du Conseil de recherches en sciences humaines du Canada (CRSH). Je tiens à remercier mon directeur de thèse, monsieur Daniel Mercure de l'Université Laval, ainsi que messieurs Christian Lalive d'Epinay et Stefano Cavalli, de l'Université de Genève (responsables de l'étude internationale CEVI).

\section{A propos de l'auteur}

Christian Bergeron, Ph.D - Université Laval

Christian.Bergeron.1@ulaval.ca

\section{Références}

Bergeron, C. (2015). Réception du 11 septembre 2001 au Québec. Persistance des mémoires nationales et émergence de mémoires globalisées. Transatlantica, 1 (à paraître, mai 2015).

Boltanski, L. (1993). La souffrance à distance. Paris : Éditions Métailié.

Chabbal, J. (2009). L'action publique à l'épreuve des indignations morales. Dans F. Cantelli; M. Roca i Escoda; J. Stavo-Debauge; L. Pattaroni (dir.), Sensibilités pragmatiques : enquêter sur l'action publique (1 ${ }^{\text {ère }}$ éd., p. 163-178). Bruxelles : Éditions Peter Lang.

Creswell, J. W. (2003). Research Design. Qualitative, Quantitative, and Mixed Methods Approaches ( $2^{\mathrm{e}}$ éd.). Thousand Oaks : Sage Publications. 


\section{CGJSC / RCESSC}

REVUE CANADIENNE

DES ÉTUDES SUPÉRIEURES

Vol. 4 - Issue 1 No. 1 - Summer / été 2015

Deschavanne, É.; Tavoillot, P.-H. (2007). Philosophie des âges de la vie. Pourquoi grandir? Pourquoi vieillir? Paris : Bernard Grasset.

Huberman, M. A.; et Miles, M. B. (1991). Analyse des données qualitatives. Recueil de nouvelles méthodes. Bruxelles : De Boeck.

Kaufmann, J.-C. (2004). L'invention de soi. Une théorie de l'identité. Paris : Hachette Littératures.

Lalive d'Epinay, C.; Concha, V.; Gastron, L.; Guichard, E.; Henriquez, G.; Lynch, G.; et Oddone, M. J. (2012). Mondialisation et mémoires de l'histoire. Une comparaison internationale et intergénérationnelle. Dans R. Bourqia (dir.), La sociologie et ses frontières. Faits et effets de la mondialisation, volume I (1 ère éd., p. 119-134). Paris : L'Harmattan.

Mannheim, K. ([1928] 1990). Le problème des générations. Paris : Nathan.

Martuccelli, D. (2006). Forgé par l'épreuve. L'individu dans la France contemporaine. Paris : Armand Colin.

Martuccelli, D. (2002). Grammaires de l'individu. Paris : Éditions Gallimard.

Radio-Canada (2010). Le bilan grimpe à 230000 morts. En ligne : http://www.radiocanada.ca/nouvelles/International/2010/02/10/004-haiti_bilan.shtml Consulté le 28 septembre 2014.

Ricœur, P. (2005). Le bon usage des blessures de la mémoire. En ligne : http://www.fondsricoeur.fr/uploads/medias/articles_pr/temoin-4.pdf Consulté le 28 septembre 2014.

Ricœur, P. (1990). Soi-même comme un autre. Paris : Éditions du Seuil.

Smith, A. ([1759] 2003) Théorie des sentiments moraux. Quadrige : Presses Universitaires de France.

Statistique Canada (2006). Recensement de 2006. En ligne : http://www12.statcan.ca/censusrecensement/2006/index-fra.cfm Consulté le 28 septembre 2014. 